\title{
Incomplete Network for Survivors of Catastrophic Illness After Release From ICUs
}

In the last 15 years, availability of beds in ICUs and new technologies, coupled with improved levels of care, have highlighted a new population of patients labeled as survivors of a catastrophic illness. These patients often require mechanical ventilation (MV) and long-term weaning procedures. Despite the fact that this group of patients represents $<10 \%$ of all ICU admissions, they account for a disproportionate burden on healthcare financial resources. Patients requiring MV occupy most beds in ICUs. A subset of these patients have weaning difficulties, so duration of MV (commonly defined as $<15 \mathrm{~d}$ ) may be abnormally prolonged. Wunsch et $\mathrm{al}^{1}$ reported that of $6,469,674$ hospitalizations in 6 American states, 180,326 (2.8\%) needed invasive MV. A total of $44.6 \%$ hospitalized patients had at least one major comorbid condition, with diabetes (13.2\%) and pulmonary disease $(13.2 \%)$ being the most common comorbidities. Only $30.8 \%$ of patients were discharged home from the hospital, while the remaining ultimately required a skilled care facility. ${ }^{1}$

Increased life expectancy has dramatically increased the number of patients who are admitted to critical care units for prolonged MV (PMV). Elderly patients have not only respiratory system insufficiency, but also multisystem insufficiency, due to numerous factors such as chronic underlying disease, infections, malnutrition, complications, invasive procedures, and medications. ${ }^{2}$

Weaning from MV that is categorized as either difficult or prolonged has a higher in-hospital mortality. ${ }^{3}$ While it is true that there is an incorrect nihilistic attitude toward this subgroup of patients, it must be noted that more than three quarters of the surviving patients are able to be liberated from a ventilator. The critical question is whether it is correct for patients who need prolonged weaning to be hospitalized in classical ICUs. ${ }^{3}$

PMV is not only a "medical" problem, but it also has an economic and social impact. In the United States, costs for MV were estimated to be $\$ 27$ billion, representing $12 \%$ of all hospital costs. ${ }^{1}$ Furthermore, about 300,000 patients per year receive prolonged life support in an ICU. This number is likely to double within a decade, with expected costs of more than $\$ 50$ billion. ${ }^{4}$

Once discharged from the ICU, these patients have a readmission rate of $67 \%$, and they spend an average of $74 \%$ of their remaining days either in a hospital or a post- acute care facility or under home healthcare. The burden of PMV also affects the families, because of financial needs, disruption of family routine, and future uncertainty. Indeed, patients who survive for one year are left with a serious burden of pervasive and persistent disability, despite aggressive care that costs a total of $\$ 38$ million. ${ }^{5}$

Once the precipitating cause of their acute episode of respiratory failure has been solved, these "chronically ill" patients are often discharged from the ICU but still require MV. Post-discharge care for patients on PMV requires transfer to a long-term care or rehabilitation facility. ${ }^{6}$ These "protected" environments for the treatment of patients requiring PMV are generically defined as chronic ventilator facilities.

Despite a lack of strong scientific evidence, specific facilities for ventilator-dependent patients do provide some clinical and financial advantages over ICU care, in terms of good prognostic outcome, tracheostomy tube removal, full rehabilitation program with better physical function recovery, better control of the emotional status, low rate of readmission, and lower costs. ${ }^{7-9}$

The main benefits of chronic ventilator facilities are the possibility of relieving congestion of ICU beds, providing a high level of nursing care, quickly responding to sudden changes in a patient's clinical condition, allowing enough time for a multidisciplinary rehabilitation approach, and acting as a bridge to home-care programs or other forms of continuous chronic assistance. However, chronic ventilator facilities have unique problems, which can be described as follows:

- Financial Reimbursement. It is difficult to compare and offer a definitive gold standard for reimbursements, due to difference between studies coming from various centers and periods of time. In fact, weaning success seems strongly affected by patient complexity and comorbidities, hospital organization and personnel expertise, availability of early physiotherapy, use of weaning management techniques, patient autonomy, and family preparation for home discharge with a ventilator. Published data are based on small samples, different clinical histories, costs based on patients coming from a single hospital, variations in care habits among different countries, differences in interventions, and equipment and 
staff involvement, thus reducing the generalization of the cost/reimbursement assessment.

- Staffing Allocation. Staffing in chronic ventilator facilities is typically characterized by the heterogeneous mix of personnel involved in a multidisciplinary approach. However, allocation of nursing time or involvement of respiratory therapists remains controversial.

- Admission and Discharge Criteria. Long-term acute care centers have emerged to provide specialized care required for PMV patients with severe malnutrition, physical deconditioning, high acuity of illness, multiple comorbidities, long hospitalizations, and frequent readmissions to acute care facilities, all of which increase risk of colonization and infection with antibioticresistant organisms. These characteristics are associated with poor long-term outcomes and high mortality rates.

See the Original Study on Page 250

In the present issue, Verceles et $\mathrm{al}^{10}$ performed a retrospective cohort study investigating the association between comorbidities and bacterial colonization status. These authors reported that patients admitted to a long-term acute care center for PMV were more likely to be transferred to an acute care facility if they had a high number of comorbid conditions and if they had infection with multidrug resistant Acinetobacter. This may suggest that colonization with multidrug resistant Acinetobacter is a marker of more severe underlying illness, or perhaps patient frailty, which would in itself trigger a necessity of transferring the patient to an acute care facility.

It has been recently shown that there is a high incidence of metallo $\beta$-lactamase producing Pseudomonas aeruginosa and Acinetobacter species in burns and surgical wards, which reflects excessive use of carbapenems and, at the same time, poses a therapeutic challenge to clinicians as well as to microbiologists. ${ }^{11}$ Interestingly, Arvaniti et $\mathrm{al}^{12}$ suggested that long hospitalization before the ICU can identify imported A. baumannii carriers, confirming the findings of Verceles et al. ${ }^{10}$ Medical patients with extended administration of antibiotics and long duration of MV in the ICU were the most vulnerable to acquisition of colonization pressure (carriers' patient-days $\times 100 /$ all patients' patient-days).

Transfer to an acute care facility was also associated with a higher Charlson Comorbidity Index. This is not surprising, even though, to our knowledge, this is the first study to show that. It would have been interesting to see which, if any, are the main comorbidities that have influenced the decision to a new "acute" admission.
The rate of achieving complete ventilator independence in specific and dedicated weaning units is generally high. It has been demonstrated that these units are cost-saving alternatives to an ICU, but only for carefully selected patients. Weaning success, however, does not in itself solve other severe issues, such as heavy financial and human burdens on families, caregivers, and health service organizations once these patients are discharged from a protected environment. ${ }^{2}$

Long-term acute care and chronic ventilator facilities play an increasingly important role in management of patients with chronic critical illness. Yet few data exist to guide decision-making about transfer or discharge. The different international medical systems need to adopt new organizational innovations and to highlight the need for a diverse program of comparative effectiveness research to determine the optimal organization of care for patients recovering from critical illness, including the best way to maximize survival and control costs for this high-risk patient group.

More research is needed on the impact of weaning facilities on outcomes and costs. In particular the following questions should be assessed: whether or not weaning facilities best operate as units within hospitals or as standalone centers; the optimum nurse, physician, and ancillary staffing; and the spillover effects of weaning facilities on critical care.

In conclusion, despite clinical and scientific evidence of costs/effectiveness for chronic ventilator facilities, their place in an organizational network and their economic recognition remain unfinished and incomplete.

Michele Vitacca MD

Rehabilitative Respiratory Division and Weaning Center Fondazione Salvatore Maugeri Istituto di Ricovero e Cura a Carattere Scientifico Lumezzane, Italy

Stefano Nava MD Pneumologia e Terapia Intensiva Respiratoria Dipartimento Cardio-Toraco-Vascolare Policlinico Sant' Orsola-Malpighi Bologna, Italy

\footnotetext{
The authors have disclosed no conflicts of interest.

Correspondence: Michele Vitacca MD, Rehabilitative Respiratory Division, Fondazione Salvatore Maugeri, Istituto di Ricovero e Cura a Carattere Scientifico, Via Giuseppe Mazzini 129, 25066 Lumezzane, Italy. E-mail: michele.vitacca@fsm.it.
}

DOI: $10.4187 /$ respcare.02262 


\section{Incomplete Network for Survivors of Catastrophic Illness After Release From ICUs}

\section{REFERENCES}

1. Wunsch H, Linde-Zwirble WT, Angus DC, Hartman ME, Milbrandt EB, Kahn JM. The epidemiology of mechanical ventilation use in the United States. Crit Care Med 2010;38(10):1947-1953.

2. Sacanella E, Pérez-Castejón JM, Nicolás JM, Masanés F, Navarro M, Castro P, López-Soto A. Mortality in healthy elderly patients after ICU admission. Intensive Care Med 2009;35(3):550-555.

3. Funk GC, Anders S, Breyer MK, Burghuber OC, Edelmann G, Heindl $\mathrm{W}$, et al. Incidence and outcome of weaning from mechanical ventilation according to new categories. Eur Respir J 2010;35(1): 88-94.

4. Zilberberg MD, Shorr AF. Prolonged acute mechanical ventilation and hospital bed utilization in 2020 in the United States: implications for budgets, plant and personnel planning. BMC Health Serv Res 2008;8:242.

5. Unroe M, Kahn JM, Carson SS, Govert JA, Martinu T, Sathy SJ, et al. One-year trajectories of care and resource utilization for recipients of prolonged mechanical ventilation: a cohort study. Ann Intern Med 2010;153(3):167-175.

6. Epstein SK, Ciubotaru RL, Wong JB. Effect of failed extubation on the outcome of mechanical ventilation. Chest 1997;112(1):186-192.
7. Byrick RJ, Mazer CD, Caskennette GM. Closure of an intermediate care unit. Impact on critical care utilization. Chest 1993;104(3): 876-881.

8. Robson V, Poynter J, Lawler PG, Baudouin SV. The need for a regional weaning centre, a one-year survey of intensive care weaning delay in the Northern Region of England. Anaesthesia 2003;58(2): 161-165.

9. Heyland DK, Konopad E, Noseworthy TW, Johnston R, Gafni A. Is it 'worthwhile' to continue treating patients with a prolonged stay (>14 days) in the ICU? An economic evaluation. Chest 1998;114(1): 192-198.

10. Verceles AC, Lechner EJ, Halpin D, Scharf SM. The association between comorbid illness, colonization status, and acute hospitalization in patients receiving prolonged mechanical ventilation. Respir Care 2013;58(2):250-256.

11. Kumar SH, De AS, Baveja SM, Gore MA. Prevalence and risk factors of Metallo $\beta$-lactamase producing Pseudomonas aeruginosa and Acinetobacter species in burns and surgical wards in a tertiary care hospital. J Lab Physicians 2012;4(1):39-42.

12. Arvaniti K, Lathyris D, Ruimy R, Haidich AB, Koulourida V, Nikolaidis $\mathrm{P}$, et al. The importance of colonization pressure in multiresistant Acinetobacter baumannii acquisition in a Greek intensive care unit. Crit Care 2012;16(3):R102. 